\title{
Structural basis for human DNA polymerase kappa (polк) to bypass cisplatin intrastrand cross-link (Pt-GG) lesion as an efficient and accurate extender
}

\author{
Vikash Jha and Hong Ling \\ Dept. of Biochemistry, Schulich School of Medicine \& Dentistry, University of Western \\ Ontario, London, ON, Canada.
}

\begin{abstract}
Cisplatin (cis-diamminedichloroplatinum) is a widely used chemotherapeutic compound. It reacts with the N7 atoms of adjacent guanines in DNA to form the Pt-1,2$\mathrm{d}(\mathrm{GpG})$ intrastrand cross-link (Pt-GG) as a major product. This bulky cisplatin adduct blocks DNA replication by replicative polymerases, thereby promoting apoptosis of rapidly dividing cancer cells. However, cancer cells have developed resistance to cisplatin, partly because of the bypass of cisplatin adducts by specialized DNA polymerases (mostly belonging to the Y-family) through translesion synthesis (TLS). The human Y-family DNA polymerase eta (pol $\eta$ ) is very efficient in replicating opposite the Pt-GG lesions, but not in extending after this adduct. Recently, it was shown that human Y-family DNA polymerase kappa (polк) is indispensable for DNA repair synthesis in dorsal root ganglion neurons exposed to cisplatin. Polא gene knockdown experiments in human cells have also indicated the involvement of polк in TLS of the Pt-GG lesion. In this study, we investigated the bypass potential and nucleotide incorporation preference of polא opposite DNA containing the Pt-GG adduct and determined two crystal structures of polk complexed with such DNA. The ternary complex structures represent two consecutive stages of lesion bypass: nucleotide insertion opposite the 5' $\mathrm{G}$ (Pt-GG2) and primer extension immediately after the lesion (Pt-GG3). Our in vitro DNA replication

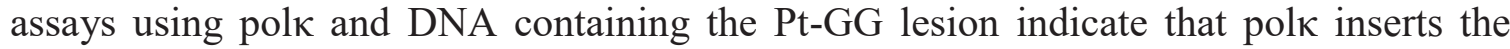
correct dCTP opposite the 5'G of Pt-GG (Pt-GG2), when a nucleotide is already inserted opposite the $3^{\prime} \mathrm{G}$ (Pt-GG1). Polא also inserts the correct dATP opposite the T base immediately after the Pt-GG (Pt-GG3). However, polк is not able to insert a nucleotide opposite the $3^{\prime} \mathrm{G}$ of Pt-GG (Pt-GG1). On the other hand, poln is very efficient in replicating the Pt-GG lesion at two insertion stages (Pt-GG1 and Pt-GG2), but the enzyme is blocked at the extension stage (Pt-GG3). In terms of fidelity, we found that polк has the fewest mis-insertions compared to two other human Y-family polymerases

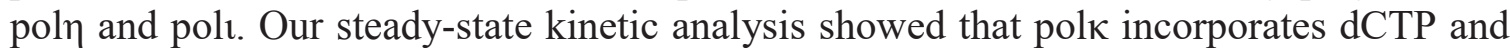
dATP opposite the 5' G of Pt-GG (Pt-GG2) and T template 5' to the lesion (Pt-GG3), respectively, with very similar relative efficiencies of $0.15-0.20$, which are only $\sim 5$ fold lower than for normal DNA replication (GG2 and GG3). Our biochemical data indicate that polk is very proficient and accurate in inserting a nucleotide after the first $G$ of the Pt-GG lesion. The structures revealed that the accuracy is achieved by stably accommodating the bases containing the cisplatin adducts in the enzyme's active site for proper Watson-Crick base pairing with the incoming nucleotide. Together, our biochemical and structural data provide the molecular basis for the accurate and

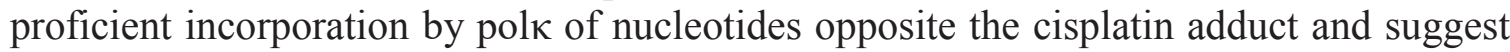
an important role of polк as a second polymerase (extender) in the efficient bypass of the Pt-GG lesion in vivo. This work holds promise for polk as a potential target for drug design, along with pol $\eta$, which together could improve the efficacy of cisplatin treatment for cancer therapy.
\end{abstract}

